Mots. Les langages du politique

\title{
Georges Frêche et ses «biographes» montpelliérains. Le défi de dire un homme
}

Georges Frêche and his Montpellier "biographers". The challenge of portraying one figure

Georges Frêche y sus "biógrafos" montpellerianos. El desafío de decir un hombre

\section{Éléonore Yasri-Labrique}

\section{(2) OpenEdition}

\section{Journals}

Édition électronique

URL : https://journals.openedition.org/mots/21594

DOI : $10.4000 /$ mots. 21594

ISSN : 1960-6001

Éditeur

ENS Éditions

\section{Édition imprimée}

Date de publication : 19 mai 2014

Pagination : $57-74$

ISBN : 978-2-84788-542-2

ISSN : 0243-6450

\section{Référence électronique}

Éléonore Yasri-Labrique, « Georges Frêche et ses « biographes » montpelliérains. Le défi de dire un homme », Mots. Les langages du politique [En ligne], 104 | 2014, mis en ligne le 19 mai 2016, consulté le 23 avril 2022. URL : http://journals.openedition.org/mots/21594; DOI : https://doi.org/10.4000/mots. 21594 


\section{Georges Frêche et ses « biographes " montpelliérains. Le défi de dire un homme}

Le 24 octobre 2010 s'éteignait Georges Frêche, ancien maire de Montpellier, plusieurs fois député de l'Hérault, président de la communauté d'agglomération de Montpellier et président du conseil régional du Languedoc-Roussillon. À sa mort comme de son vivant, les polémiques à son sujet se sont multipliées, et il est généralement admis que peu d'hommes politiques français n'ayant jamais exercé de fonctions nationales ont fait couler autant d'encre. Longtemps membre du Parti socialiste, il est exclu de cette formation le 27 janvier 2007. Dans la foulée de cet événement retentissant, trois livres lui sont, pour la première fois, entièrement consacrés, et ce à quelques mois d'intervalle : Georges Frêche, la sagesse de la démesure de François Delacroix (avril 2007), Georges Frêche, grandes heures et décadence de Karim Maoudj (mai 2007) et L'Assassinat raté de Georges Frêche d'Alain Rollat (février 2008). Le premier de ces auteurs a été pendant plus de 25 ans l'un de ses proches collaborateurs, les deux autres sont journalistes. C'est donc sur les deux derniers ouvrages que portera l'essentiel de notre analyse tandis que le premier texte nous servira de référence d'appoint.

Pourquoi cette «trilogie» est-elle parue à ce moment-là? Quels sont les enjeux discursifs, politiques et médiatiques de cette mise en scène à répétition? Quelle grille de lecture nous offre-t-elle de la carrière de cet homme controversé? Quels sont les modes d'écriture privilégiés dans ces productions discursives? Autant d'interrogations que notre contribution, au carrefour des sciences de l'information et de la communication, du langage et du politique se propose d'explorer. Après avoir présenté notre corpus, nous tenterons de repérer les parcours textuels qui caractérisent les deux principaux ouvrages et de les définir. Correspondent-ils à des enquêtes journalistiques sur l'histoire d'un cheminement politique? Sur quelles sources s'appuient-ils? Vers quelle relecture des discours médiatiques antérieurs nous orientent-ils? Sortent-ils du cadre du récit de vie pour s’apparenter à un portrait? Présentent-ils alors des lignes de convergence ou de divergence sur le plan discursif? Et quelle figure du professionnel du journalisme politique contribuent-ils ainsi à construire?

Université Montpellier 3 (Dipralang, EA 739)

eleonore.yasri@univ-montp3.fr 


\section{Entre unicité et disparité : un corpus singulier}

Les ouvrages que nous avons choisi d'étudier semblent répondre aux exigences d'une forme de dramaturgie classique : la loi des trois unités est globalement respectée. L'unité de lieu avec pour référence la ville de Montpellier, et plus largement la région Languedoc-Roussillon, fonctionne à un double niveau : c'est à la fois le point d'ancrage des trois auteurs, l'espace physique ou symbolique d'où émerge leur projet d'écriture et l'endroit géographique où s'inscrivent les principaux événements de leur récit. L'unité de temps, certes envisagée avec beaucoup de souplesse dans son extension, se retrouve dans chacun des ouvrages : elle a pour référent chronologique une période de 30 ans qui s'étale entre le 25 mars 1977, date de l'accession de Georges Frêche à la mairie de Montpellier et le 27 janvier 2007, jour de son exclusion définitive du Parti socialiste français. Le point de départ et le point d'arrivée prennent une importance variable sous la plume de chaque auteur mais ils encadrent et légitiment les différents parcours textuels. Quant à l'unité d'action, elle correspond à la carrière politique de Georges Frêche en tant que maire puis président de conseil régional, une carrière marquée par une succession de faits formant la trame de chacun des trois ouvrages.

C'est cette apparente unicité qui nous a poussée à analyser ce corpus auquel s'applique, par ailleurs, pour reprendre les termes d'Yves Agnès (2002)1 " «la loi de proximité ». En effet, les quatre axes qui la constituent sont ici clairement représentés : les axes géographique, chronologique et sociétal, comme nous venons de le voir, mais aussi l'axe psychoaffectif dans la mesure où, même si les trois auteurs sont en désaccord sur la manière dont cela s'est produit et sur les résultats obtenus, tous pointent l'abolition des distances entre l'élu et les électeurs. Sur son terrain, l'action publique de Georges Frêche a redessiné la relation entre l'homme politique et ses concitoyens, qui se caractérise par ce que Christian Le Bart appelle «l'inversion des stigmates» :

Le refus de confier par exemple les pouvoirs locaux à des autorités élues au plus près des populations [...] témoigne tout au long du $19^{\mathrm{e}}$ siècle de cette méfiance. élu par le conseil, le maire deviendrait prisonnier des intérêts locaux les plus étroits. Pour bien administrer la commune, il lui faut tenir son autorité d'ailleurs, d'en haut, de l'État synonyme de rationalité et de progrès. [...] Ce modèle de légitimation a fonctionné pendant deux siècles; il est aujourd'hui grippé. Crise de l'ÉtatProvidence? Crise de la représentation? Les stigmates se sont inversés, la distance est à présent suspecte et la proximité légitime. (Le Bart, 2005, p.13-14)

1. «Dans son Manuel de journalisme, Yves Agnès décline la loi de proximité selon quatre axes: proximité géographique, chronologique, psychoaffective et sociétale. Ces quatre entrées s'articulent sur un noyau central qui est la figure du lecteur dont on évalue le potentiel d'intérêt pour l'information en fonction de ce qui lui est proche.» (Cité par Ringoot et Rochard, 2005, p. 74.) 
Dans son livre intitulé Georges Frêche, la sagesse de la démesure, publié aux éditions Alter Ego et paru le 15 avril 2007 (deux mois et demi après l'éviction de Georges Frêche du PS par la commission nationale des conflits de cette formation), François Delacroix, l'un de ses collaborateurs les plus fidèles, alors directeur général des services de la communauté d'agglomération de Montpellier, parle même d'une « histoire d'amour entre Georges Frêche et Montpellier ». Sur la quatrième de couverture, il explique que son «propos est d'essayer de mieux faire comprendre l'alchimie de la réussite du développement de Montpellier et de son agglomération en survolant ces vingt-cinq dernières années, non pas chronologiquement mais en traitant des sujets propres à cette ville et à la personnalité de Georges Frêche. „ Certes partisan, cet ouvrage se veut aussi éclairé et surtout inscrit dans la réalité locale : rédigé en Languedoc-Roussillon, diffusé par un éditeur de Céret (Pyrénées-Orientales). Il n'établit pas de lien direct avec l'actualité politique.

À peine six semaines plus tard paraît un deuxième ouvrage qui a pour titre Georges Frêche, grandes heures et décadence et qui, bien que publié aux Éditions de Paris, assume à son tour un ancrage local : il émane en effet d'un journaliste du Midi Libre qui s'interroge essentiellement sur l'homme et sa carrière en contexte montpelliérain. L'ouvrage, dont la couverture ivoire s'orne d'une caricature du président du conseil régional, est présenté au dos comme «une enquête nourrie de nombreux témoignages et un travail d'analyse que jamais personne n'avait voulu engager jusque-là ». Les différentes procédures de présentation du texte convergent toutes dans le même sens : elles signalent que ce travail journalistique s'apparente à celui d'un critique qui cherche à dénoncer plutôt qu'à encenser. Karim Maoudj mentionne qu'il a mûri ce projet d'enquête durant plus de 15 ans : il n'y aurait de sa part aucune intention initiale de faire référence à «l'air du temps »².

Pour répondre davantage au détracteur qu'à l'admirateur, Alain Rollat, journaliste né à Montpellier, diplômé de l'École supérieure de journalisme de Paris, qui a fait ses débuts au Midi Libre et a longtemps travaillé pour Le Monde avant de fonder La Gazette de Sète, publie en février 2008 un essai aux Éditions Singulières de Sète : L'assassinat raté de Georges Frêche. L'auteur, qui a consacré plusieurs publications à des hommes politiques³, n'en est pas à son coup

2. «Une autre entrée dans l'ethnosocioculture “moderne" concerne l'imaginaire le plus contemporain (d'aucuns diraient le plus “branché”), le plus instable aussi : ce qu'on appelle communément "l'air du temps" [...]. Cette partie [...] de la CESC [compétence ethno-socio-culturelle] révèle, pour un moment donné, les valeurs auxquelles adhère (ou proclame adhérer) une société, son atmosphère idéologique, ses obsessions, ses peurs, ses rêves, ses engouements... collectifs. Elle se manifeste de manière beaucoup plus explicite (spectaculaire même) que les autres composantes de cette même CESC, en particulier dans les médias. » (Boyer, 2003, p. 29)

3. Les hommes de l'extrême droite. Le Pen, Marie, Ortiz et les autres, Paris, Calmann-Lévy, 1985; Ce terrible monsieur Pasqua, Paris, Orban, 1988 (avec Philippe Boggio); Tjibaou le Kanak, Lyon, La Manufacture (L’Histoire partagée : Biographie), 1989; Delors, Paris, Flammarion, 1993. 
d'essai. De plus, dès novembre 2007, il avait publié au Seuil un livre de conversations avec Georges Frêche : Il faut saborder le PS. Ici, la quatrième de couverture fait à la fois clairement référence aux turbulences politiques de l'époque et aux devoirs du journaliste en matière d'information :

Alain Rollat se penche sur le cas Georges Frêche, ancien maire de Montpellier, président du conseil régional du Languedoc-Roussillon, exclu du Parti socialiste à la suite de plusieurs dérapages verbaux. Comment le maire de «Montpellier la Surdouée», dont l'auréole politique était celle d'un bâtisseur flamboyant, est-il brusquement devenu, sous le regard des médias, un détestable potentat taxé de racisme? L'image de ce notable controversé reflète-t-elle la vérité vraie ou n'en est-elle qu'une expression déformée? Pour en avoir le cœur net, Alain Rollat passe cette image au crible des «détecteurs de mensonges» mis au service du journalisme moderne par les sciences de la communication.

En nous intéressant principalement aux livres sur Georges Frêche des journalistes Karim Maoudj et Alain Rollat, nous souhaitons apporter des éléments de réponse à nos différentes interrogations afin de caractériser les parcours textuels de ces ouvrages qui se revendiquent comme le fruit d'un travail journalistique où l'investigation, le recueil des témoignages et l'analyse des données jouent un rôle primordial. À quelles contraintes de genre répondentils? Comme le note Jean-Michel Adam (2001, p.10), cette question «est une question linguistique majeure» dans la mesure où «la catégorisation générique même vague d'un objet discursif en permet la production autant qu'elle en guide la lecture ». Or, définir génériquement ces livres de journalistes politiques qui ont le projet de «dire Georges Frêche » constitue un défi. D’emblée, il s'avère difficile de les classer parmi les biographies car la complexité des intentions affichées par les auteurs et celle des réalisations effectives se révèle très vite au lecteur qui entrevoit alors des parcours textuels superposés, entre monographie, reportage et galerie de portraits.

\section{Des monographies objectives?}

Les trois textes analysés se présentent comme des récits, non pas directement de la vie de Georges Frêche, mais de ses actions en politique pour François Delacroix et Karim Maoudj, et des répercussions de ces actions pour Alain Rollat. Il ne s'agit donc pas de biographies à proprement parler mais plutôt de monographies, dans la mesure où chacun de ces ouvrages s'attache à offrir une lecture circonstanciée de la carrière de celui qui, après 27 ans passés à la tête de la mairie de Montpellier, ne l'a quittée que pour occuper la présidence du conseil régional du Languedoc-Roussillon. Le terme «monographie», emprunté au vocabulaire des sciences humaines, est à entendre ici dans son acception sociologique telle qu'elle est indiquée dans le Trésor de la langue française 
informatisé4 : «Enquête ou étude approfondie limitée à un fait social particulier et fondée sur une observation directe qui, mettant en contact avec les faits concrets, participe de l'expérience vécue et relève de la sociologie compréhensive (d'apr. Foulq. Sc. soc. 1978). »5

En effet, ces narrations ne sont pas prioritairement basées sur des récits antérieurs - écrits ou oraux - des événements marquants de la vie de Georges Frêche, mais bien sur des interactions réelles, en contexte pour François Delacroix en tant que collaborateur ainsi que pour Karim Maoudj en tant que journaliste de terrain au Midi Libre, ou a postériori en ce qui concerne Alain Rollat qui, dans le but initial de publier un livre d'entretiens ${ }^{6}$, interviewe longuement l'élu local après son exclusion du PS. Dans tous les cas, l'objectif annoncé des auteurs est de chercher à comprendre et de faire comprendre au public qui est vraiment cet homme fréquenté en diverses occasions et finalement partie intégrante de leur existence même.

\section{D’une subjectivité revendiquée... à une objectivité recherchée}

La volonté de distanciation est cependant variable d'un auteur à l'autre. L'expression de sa subjectivité est hautement revendiquée par le fidèle collaborateur qu'est François Delacroix :

J'ai ressenti l'envie plutôt que la nécessité, d'écrire un ouvrage expliquant l'alchimie de cette longévité aux côtés d'un homme réputé « difficile à vivre». Pour que les choses soient claires, je souligne être parfaitement conscient que l'envie participe de l'affect, tandis que la nécessité est du domaine de l'intellect. Oui, Georges Frêche est « impossible» [...]. Mais, pour moi, il est avant tout un homme attachant. (F. D., p. 13)7

À l'inverse, les deux journalistes, même s'ils reconnaissent ne pas pouvoir aller à l'encontre de leur sensibilité, font vœu d'objectivité. Le point de vue de Karim Maoudj, qui catégorise son ouvrage comme une «enquête journalistique » puis comme un «essai », c'est-à-dire un texte où s'inscrivent à la fois le report des faits et un point de vue critique, s'ancre dans sa connaissance, sa pratique et sa perception du terrain :

Cette enquête journalistique est avant tout un essai. Elle exprime un regard. Forcément différent, parce que sans complaisance. Elle raconte un parcours. Elle rafraîchit les mémoires. Elle se veut [...] un éclairage pour comprendre la cité et son ancien maire. [...] Ce travail vise à donner les clés d'une histoire où les acteurs sont intimement

4. http://atilf.atilf.fr/dendien/scripts/tlfiv5 (article « monographie » consulté le 19 janvier 2013).

5. Référence : Foulquié, 1978.

6. Georges Frêche, Il faut saborder le PS, Paris, Le Seuil, 2007 (Conversations avec Alain Rollat).

7. Les citations détachées, extraites du corpus, sont suivies des initiales de l'auteur et du numéro de page. 
imbriqués. Bien sûr, il faudrait être de mauvaise foi pour ne dresser qu'un bilan négatif de l'action de Georges Frêche à Montpellier. Son empreinte est incontestable, tout comme sa réussite. Mais cela ne saurait suffire à tout expliquer. (K. M., p.9-10)

Quant à Alain Rollat, il s'interroge constamment sur les liens entre information et vérité : il cherche à offrir un « récit de cette recherche » et un « exemple pédagogique» (p.13) à ses lecteurs, et donne au début de son argumentation les indications suivantes sur sa démarche :

En ce mois de février 2006, j'ai ainsi pour le «roi Georges» - je le reconnais - le préjugé favorable que m'a inspiré l'observation de ses activités politiques et de ses réalisations dans sa capitale régionale. Mais je ne suis pas dupe de mes penchants. J'ai l'habitude de garder mes sentiments à distance de ma plume. [...] Je me pose les questions prévues par la procédure journalistique applicable en la matière. (A. R., p. 21)

Dans les deux cas, le vœu d'objectivité est contrebalancé par une absence affichée de neutralité subjective. Karim Maoudj et Alain Rollat s’inscrivent dans un genre discursif où le sujet d'énonciation prend position explicitement et énonce des questionnements, mais aussi des opinions. Au-delà du récit circonstancié et argumenté se dessine un jugement évaluatif dont les enjeux axiologiques dépassent le cadre de la déontologie journalistique. Le journaliste devenu auteur s’affranchit ainsi de certaines contraintes imposées à la profession.

\section{Construction d'un ethos professionnel}

Il nous semble important de noter malgré tout que les livres de Karim Maoudj et Alain Rollat se distinguent par une revendication commune : leur rattachement à la sphère journalistique comme gage de fiabilité et de sérieux. Tous deux n'assument pas seulement leur appartenance au monde des médias - en l'occurrence sous leur forme la plus traditionnelle, la presse écrite, dont les caractéristiques sont notamment rappelées par Charaudeau (1997, p. 125) ${ }^{8}$-, ils se présentent surtout comme des professionnels respectables pour qui le métier de journaliste suppose une déontologie stricte qu'ils défendent avec ferveur et dont ils se portent garants en ce qui les concerne. Ainsi, comme le montrent les deux citations précédentes, ils ne prétendent pas proposer des récits objectifs, ce qui ne serait nullement crédible, mais le résultat de recherches menées selon les règles de l'art, placées sous le sceau de l'honnêteté intellectuelle et morale. Comme l'indique Roselyne Koren :

8. «Un rapport distancié entre celui qui écrit et celui qui lit, du fait de l'absence physique de l'instance d'émission et de l'instance de réception, l'une vis-à-vis de l'autre; une activité de conceptualisation de la part des deux instances pour se représenter le monde, ce qui produit des logiques de production et de compréhension spécifiques; un parcours oculaire multi-orienté de l'espace d'écriture qui fait que ce qui a été écrit reste comme une trace sur quoi on peut constamment revenir : celui qui écrit pour rectifier ou effacer, celui qui lit pour remémorer ou recomposer sa lecture.» 
La neutralisation de la prise de position serait dans ce cas un mode de protection contre le risque de l'engagement idéologique militant, mais serait compatible, aussi paradoxal que cela puisse paraître, avec l'exercice d'une fonction critique qui a pour enjeu l'évaluation de la vérité et de la validité des dires des hommes politiques. (Koren, 2004, p. 20)

Leur «profession de foi » relève, ainsi que le soulignerait Dominique Maingueneau (2005, p.71)9, d’une "scénographie partagée» mais contribue ce faisant à construire une certaine figure du professionnel du journalisme politique, oscillant entre discours informatif et discours critique dans le but de donner au lecteur des clés pour interpréter les données et se forger sa propre opinion. Ainsi, en ouverture de leur ouvrage ou tout au long du récit, l'autoportrait des rédacteurs apparaît en filigrane, à travers la construction de leur ethos (Amossy, 2000 et 2010). Mais il est à noter que chacun, en tant qu'auteur indépendant, entre également en conflit avec cet ethos journalistique : Alain Rollat en s'impliquant directement à travers l'emploi du pronom «je », Karim Maoudj de manière implicite en superposant à la première personne du singulier le pronom « elle» désignant son enquête.

\section{Des postures journalistiques opposées}

En tant que professionnels de l'information, les deux hommes se livrent à un double reportage : le recueil à la source de faits d'actualité et la relation minutieuse des résultats de leurs investigations. Comme le rappellent Roselyne Ringoot et Yvon Rochard (2005, p. 79), « le reportage doit transporter le lecteur dans l'action par la médiation du journaliste, aussi sa propre mise en scène est-elle incontournable». Par leur position de journalistes, et donc d'enquêteurs de terrain, les deux auteurs vont donner à voir aux lecteurs une réalité à laquelle ils ont pris effectivement part d'une manière concrète. Pourtant, bien que se réclamant de la même discipline, Karim Maoudj et Alain Rollat proposent des récits qui se différencient notamment sur deux points : le rapport à l'actualité et l'utilisation des témoignages.

\section{«Raconter Georges Frêche » : le projet de Karim Maoudj}

Karim Maoudj indique dans son avant-propos qu'il a rencontré Georges Frêche pour la première fois en septembre 1989, rencontre difficile qui a suscité en lui questions et incompréhensions. Il explique comment, sa curiosité et sa colère ainsi attisées, il a envisagé de rédiger cet ouvrage :

9. «La scénographie est ainsi à la fois ce dont vient le discours et ce qu'engendre ce discours; elle légitime un énoncé qui, en retour, doit la légitimer, doit établir que cette scénographie dont vient la parole est précisément la scénographie requise.» 
L'idée de raconter Georges Frêche est née, dans mon esprit, il y a fort longtemps. Elle repose sur une seule et unique ambition : remettre les choses à leur place, rendre à César ce qui lui appartenait. En un mot, essayer d'expliquer la réalité de près de trente ans de pouvoir politique d'un homme, qui n'a cessé d'imposer une chape de plomb morale et psychologique sur une ville qui n'a pas su échapper à ses griffes. (K. M., p. 9)

La date exacte du début du processus de maturation n'est pas précisée, mais tout laisse à entendre que l'accumulation des informations, l'interprétation des données puis la rédaction elle-même du récit se sont étalées sur de nombreuses années et ne sont aucunement en lien avec l'actualité récente. Certes, la densité de la relation, d'ordre thématique et non pas chronologique, penche en faveur d'un travail de longue haleine. Les deux moments phares du récit, 1977 et 2007, amplement cités, permettent également de penser à un livre anniversaire.

Les allusions aux récentes affaires sont limitées. À peine effleurées en introduction, elles ne sont véritablement abordées que dans le troisième chapitre, à commencer par l'exclusion de Georges Frêche du PS qui ne fait l'objet que de quelques mentions presque anecdotiques. Les deux événements déclencheurs, désignés sous les termes «l'affaire des harkis» (février 2006) et «l'affaire des “Blacks” de l'équipe de France de football » (janvier 2007) occupent un paragraphe en fin de chapitre. Un autre passage est consacré à la première d'entre elles dans le chapitre 5 (p.137), et une allusion supplémentaire est faite lorsqu'il s'agit d'attaquer le traitement de l'information par La Gazette de Montpellier (p.161). C'est peu. Et il est alors possible de faire remarquer naïvement la coïncidence heureuse de la parution de l'ouvrage avec la campagne de médiatisation nationale qui a eu lieu autour de Georges Frêche après son bannissement politique.

À vrai dire, ce n'est pas la seule concomitance fortuite : le livre de Karim Maoudj n'a-t-il pas paru moins de deux mois après celui de François Delacroix? Ouvrage orienté dans le sens inverse mais fonctionnant de la même manière : sans respecter la chronologie, en mettant en valeur l'action et la personnalité de l'ancien maire de Montpellier, en survolant les trente dernières années. Les ressemblances discursives entre les deux livres sont frappantes : construction, thématiques, mise en valeur de la notion de témoignage direct... De plus, les mêmes anecdotes sont reprises, formant un réseau de résonances intertextuelles significatif, comme le montre l'exemple concernant la préparation des élections municipales de 1977 :

C'est Michel Lacave [...] qui est alors en charge du dossier. Il préside, en effet, l'association Citoyens et urbanisme, mise sur orbite en avril 1975 par le candidat pour réfléchir aux meilleures possibilités de développer Montpellier. [...] Le travail réalisé donne naissance à un ouvrage, vendu 6 francs à l'époque, et présenté sous la forme d'une Lettre ouverte aux Montpelliérains. Au passage, on note que c'est la 
première fois qu'un candidat se dote d'autant de moyens d'information et de communication pour s'adresser aux électeurs. (K. M., p. 98)

Pour l'organisation de celle-ci, Georges Frêche crée une association «Citoyens et urbanisme», présidée par l'universitaire Michel Lacave [...]. Cette association invite la gauche «moderne» dans des conférences pour débattre avec les citoyens de sujets nouveaux, publie un document-programme vendu 6 francs chez les buralistes [...]. Cette association fut une véritable machine de guerre pour prendre la ville. (F. D., p. 37)

Malgré toutes ces similitudes et la quasi-simultanéité de la parution des deux livres, le fait qu'ils soient vraisemblablement sortis en lien avec l'actualité reste tacite. Par ailleurs, Karim Maoudj ne cite jamais l'ouvrage de son prédécesseur mais, vu les délais, il se peut que son livre ait déjà été soumis à l'éditeur au moment où il était distribué. Toutefois, le nom de François Delacroix est mentionné à deux reprises (p.135 et p.149) et une allusion voilée au titre de son opus laisse planer un doute : «On choisit de combattre le risque par la démesure» (p.106). Quoi qu'il en soit, les deux ouvrages, auxquels ne tardera pas à s'ajouter L'assassinat raté de Georges Frêche, fonctionnent en circuit fermé : un livre appelle un autre livre, et les réponses successives en viennent finalement à former une construction médiatique en boucle.

Par ailleurs, le livre de Karim Maoudj, journaliste régional ayant couvert l'actualité de Georges Frêche entre 1989 et 2007, s'appuie également sur d'innombrables témoignages rapportés, qu'ils soient antérieurs à sa prise de fonction au Midi Libre ou contemporains. Comme indiqué sur la quatrième de couverture, cette notion de témoignage est prépondérante et le recours à ce que d'autres ont observé doit venir augmenter encore la crédibilité de l'auteur. Le premier paragraphe du premier chapitre s'ouvre ainsi sur des paroles de Georges Frêche, prononcées en 1970 et dont il est suggéré que c'est le secrétaire de la section socialiste de Montpellier, Michel Crespy, qui les a diffusées. Le second paragraphe débute par une anecdote, de 1967, «rapportée à l'auteur par un éminent universitaire montpelliérain» (p.14), dont le nom ne sera jamais mentionné. À partir de là, les références anonymes se multiplient. D’un bout à l'autre du livre, les sources sont indiquées par leur statut : « professeur» (p.17), «ancien adjoint» (p. 28), «commerçant» (p.32), « responsable syndical» (p.34), et ainsi de suite tout au long des différents chapitres - et même, vers la fin, sous des dénominations encore plus floues : «un acteur de la vie économique locale» (p.173), « un acteur extérieur à l'état-major» (p.178), «un ancien proche collaborateur» (p.179) - et rarement par leur identité. Ainsi, malgré une volonté de mise en perspective historique des événements, la traçabilité des témoignages est rendue difficile et leur rôle de gage de fiabilité est considérablement affaibli, un flou informationnel qui porte atteinte aux principes éthiques du journalisme. 


\section{Retrouver une image : la quête d'Alain Rollat}

Alors que les ouvrages de François Delacroix et de Karim Maoudj présentent, dans leur structure, de multiples similarités, l'opus proposé par Alain Rollat est nettement différent. D’abord par son angle d'approche : il ne s'agit pas de rédiger un hommage ou une critique à la suite d'une enquête de plusieurs années et qui correspondrait à un « anniversaire », mais bien de s’inscrire dans les débats de l'époque. Certes, au cours de cette enquête, les principaux événements de la carrière de Georges Frêche sont évoqués comme dans les livres précédents sans que la chronologie soit respectée et en fonction des différentes thématiques abordées, mais ce qu'Alain Rollat propose au lecteur, c'est avant tout un "arrêt sur image » au moment de «l'affaire des harkis» qui correspond, selon lui, au point de départ du lynchage médiatique de l'ancien maire de Montpellier. Le journaliste ne prétend pas vouloir remonter le temps suivant un projet de longue date; il s'inscrit au cœur de l'actualité et se livre à un travail d'analyse sur le rôle de la presse en politique, comme en témoignent les très nombreuses formes interrogatives qui sillonnent les quatre chapitres de l'ouvrage. L'actualité, ce sont donc bien sûr les «affaires» de 2006 et 2007 et l'exclusion de Georges Frêche du PS. Mais pas seulement. Dès l'introduction, ces faits sont clairement mentionnés, mais précédés de ce paragraphe :

De retour sur ma terre natale, après mon départ du Monde en 2001, j’ai donc été surpris de constater à quel point l'image que j'avais conservée de Georges Frêche ne correspondait plus à celle qui était véhiculée par la plupart des médias régionaux et nationaux, y compris par Midi Libre et Le Monde, mes deux points d'ancrage professionnel. J'avais gardé du maire de “Montpellier la surdouée” l'image d'un bâtisseur visionnaire, je me retrouvais confronté à l'image d'un potentat féodal. (A. R., p.12)

L'actualité, «l'air du temps», c'est tout autant pour lui l'ensemble des discours circulants, médiatiques, comme la référence aux deux quotidiens l'indique, mais aussi «livresques» dans la mesure où les deux images avancées à la fin de cette citation correspondent, d'une part, au message de François Delacroix, d'autre part, à la vision de Karim Maoudj. Alain Rollat ne fait ici qu'une allusion voilée à ses collègues auteurs, mais leur évocation sera tout à fait explicite dans la deuxième moitié de l'ouvrage. Leurs textes seront cités et commentés avec équité à plusieurs reprises dans le chapitre 3 mais, dans le suivant, seul le journaliste du Midi Libre sera de nouveau mentionné et ce, sur un ton beaucoup plus caustique, notamment à propos de ses propres rapports à la presse locale :

Dans son ouvrage sur «Georges Frêche, grandes heures et décadence», Karim Maoudj, en toute bonne conscience, consacrera tout un chapitre, bourré d'erreurs factuelles et d'interprétations partisanes, à «La Gazette de Montpellier ou l'allé- 
geance au pouvoir» sans consacrer la moindre ligne aux jeux de pouvoir de Midi Libre, son propre journal... il y a un journalisme de l'à-peu-près. (A. R., p. 145)

Il apparaît ainsi que l'intention d'Alain Rollat est de faire de son ouvrage une réponse circonstanciée à celui de son confrère ayant esquissé de Georges Frêche une figure de tyran. Mais sur un mode très différent : focalisation sur les développements récents et non pas sur le long terme; modalité interrogative primordiale et non pas priorité à la modalité assertive...

Une autre différence réside dans le traitement des témoignages. Tout comme son collègue, Alain Rollat s'appuie sur de nombreuses sources extérieures. L'une d'entre elles est Georges Frêche lui-même avec qui il s'est longuement entretenu avant de produire cet ouvrage. D'autres sont constituées de paroles de journalistes, Alain Rollat ayant d'emblée annoncé sa volonté de décortiquer les discours médiatiques. Mais la plupart des collègues qu'il cite conservent un anonymat de façade, démarche justifiée ainsi dans une note de bas de page :

Le propos de l'auteurvisant à décrypter le fonctionnement d'un processus, et non à mettre en cause des responsabilités individuelles, c'est délibérément que certains des écrits cités dans cet ouvrage ne seront rapportés que sous les initiales de leurs auteurs afin de ne pas personnaliser la controverse. (A. R., p. 22)

À l'inverse de Karim Maoudj qui ne commente pas pourquoi il maintient dans l'anonymat le plus complet ses multiples sources, Alain Rollat laisse une porte à l'identification des témoins impliqués en assumant son attitude par un refus d'entrer dans des querelles de personnes au sein de la profession. Notons que cette discrétion n'est pas systématique puisque plusieurs confrères sont nommément cités tels Jacques Molénat (p.79) ou André Laurens (p.111). Par ailleurs, ses sources issues d'autres milieux que le journalisme, assurément tout à fait secondaires, sont clairement nommées, par exemple Daniel Boulet (p. 108).

Les ouvrages des deux journalistes présentent donc des divergences très nettes qui rendent malaisée la caractérisation du genre discursif auquel ils appartiennent : les positionnements différents, les mises en récit personnalisées, les jeux d'opacité ou de transparence dans les références, invitent à une réflexion sur l'absence d'unicité narrative. Qu'en est-il des aspects descriptifs des personnages?

\section{Portraits ou caricatures?}

La rubrique «portrait» occupe une place importante au sein des différents organes de presse et il est raisonnable de penser qu'un ouvrage consacré à une personnalité politique accorde une part appréciable à ce mode d'expression dont «l'objectif [...] est de mettre en scène une personne choisie, mais la 
présence induite du journaliste est tout aussi importante: marques évaluatives dans les descriptions, ambiance, imbrication de citations renvoyant à l'entretien... » (Ringoot, Rochard, 2005, p. 78-79).

\section{Georges Frêche : le Personnage}

Le héros de ces trois livres est sans conteste Georges Frêche ${ }^{10}$, dont la vie privée n'est pas (ou à peine) évoquée, mais dont la personnalité et la carrière sont largement revisitées. Comme tout homme public, ce n'est pas seul qu'il a pu mener son action politique et le lecteur s'attend donc à une galerie de portraits croquant les membres de l'entourage du personnage principal. C'est en effet ce à quoi se livre François Delacroix dans la deuxième partie de Georges Frêche, la sagesse de la démesure, qu'il consacre entièrement à tous ceux qui ont « fait » ce que Georges Frêche est devenu. À travers plusieurs chapitres, il anime toute une série d'esquisses permettant aux lecteurs de se représenter ses soutiens indéfectibles ou ses opposants, tous inscrits dans une configuration politique locale. Ces personnages se retrouvent dans les deux livres de journalistes, mais leur portrait, encore précis chez Karim Maoudj, s'estompe véritablement chez Alain Rollat. Dans les deux cas, détaillés ou à peine ébauchés, ils paraissent anecdotiques et relégués à l'arrière-plan des récits, Georges Frêche occupant à lui seul l'avant-scène, un Georges Frêche omniprésent et multidimensionnel dont la personnalité complexe est finalement mise en abîme

C'est sans surprise que le portrait de Georges Frêche envahit l'espace discursif des ouvrages qui lui sont consacrés. Chacun des auteurs semble vouloir éviter la schématisation, qu'elle soit positive ou négative, même François Delacroix qui, dès son introduction et sans cacher son affection pour le personnage, en affirme les deux facettes sur le registre de la concession: "Oui, Georges Frêche est “impossible”, exigeant avec ses collaborateurs, provocateur et parfois colérique. Oui, il a bien d'autres défauts et autant, sinon plus encore, de qualités» (p.13). De son livre hommage, il ressort cependant une vision extrêmement favorable à l'ancien maire de Montpellier, sans doute bien résumée dans cette formule inédite : un «amoureux des Lumières » (p. 259), et un éloge du frêchisme en tant que «profonde conviction que l'avenir des sociétés s'écrit seulement si les civilisations font preuve d'altérité » (p. 263).

\section{Karim Maoudj : esquisse d'un portrait «frêchien »}

Ce portrait hagiographique ne se retrouve sous la plume d'aucun des deux journalistes. Dans l'ouvrage de Karim Maoudj, c'est une image beaucoup moins

10. Voir aussi à ce propos le film Le Président, documentaire réalisé parYves Jeuland sur la dernière campagne électorale de Georges Frêche et sorti le 15 décembre 2010. 
sympathique qui apparaît dès l'ouverture, celle d'un homme ayant le «sens de l'intrigue et du complot» (p. 21), qui « joue le bulldozer» (p. 31), dont la « mégalomanie» (p.35) est insupportable, qui se conduit «tel un monarque» (p.40), ces notations construites en crescendo emplissant le premier chapitre. Par la suite, de manière plus ambiguë, il sera qualifié de «romantique» (p.44), d'«inclassable» (p.57)... mais l'ambiguïté disparaît assez vite au détour de litotes («il n'est pas guidé par un excès de morale politique», p. 88) ou d'hyperboles («le nouveau maire assure sa véritable entrée en piste. Elle est, à la manière de l'homme, grandiose, monumentale», p. 108). L'auteur lui reconnaît du «charisme » et du «charme » (p.130), mais cette tendance au contraste s'effrite rapidement et les critiques répétitives se multiplient : «son désir de contrôle des hommes et des femmes est entier» (p.136), «il n'y a pas de limites dans la volonté de Georges Frêche de contrôler ses adjoints» (p.144), «son champ de servilité ne se limite pas aux seuls élus. La tentative de féodalisation des hommes et des femmes n'a, en effet pas de limites» (p. 145)... L'accélération des attaques ainsi que la reconduction lexicale transforment petit à petit le portrait sévère mais d'intention réaliste en une caricature, c'est-à-dire une «image non conforme à la réalité qu'elle représente ou suggère, et par rapport à laquelle elle est une altération déplaisante ou ridicule ${ }^{11}$. Celle-ci est renforcée à travers l'usage de l'adjectif « frêchien » dont la première actualisation offre une définition prototypique : "à la manière "frêchienne", c'est-à-dire brutale et sans appel» (p.146), et justifiée par la personnalité même du héros devenu antihéros : "Georges Frêche est l'illustration parfaite, jusqu'à la caricature, de ce type de comportements, adossé à la féodalisation [...] des acteurs d'une cité» (p.155). Vu sous les traits d'un monarque, l'homme est confirmé dans sa figure de tyran dont le « despotisme» (p.168) est constamment dénoncé.

Reconnu « personnage clé de l'histoire de Montpellier» (p.171), il a droit en fin de parcours textuel à une ligne rétrospective tout à fait élogieuse : « L’homme est jeune, érudit, intelligent, diplômé et, surtout, disposant d'une vision généreuse pour Montpellier » (p.171). Elle arrive tardivement et n'efface pas, malgré d'autres expressions valorisantes parcimonieusement distribuées sur les dernières pages, les traits difformes que les phrases de l'auteur ont tracés tout au long des pages précédentes, d'autant plus que cette vision flatteuse date de 1977 et est condamnée à être gommée. Le portrait que Karim Maoudj présente n'est pas une image figée, un tableau achevé. C'est, à l'instar du portrait de Dorian Gray sous la plume d'Oscar Wilde, une peinture en perpétuelle transformation à laquelle chaque jour ou chaque événement apporte une couche supplémentaire de vieillesse, d'arrogance, de mépris, de laideur, d'usure. La description de cette personnalité, c'est en fait ce que laissait entendre le titre programmatique de l'ouvrage, clin d'œil à Balzac, à savoir le récit d'une

11. http://atilf.atilf.fr/dendien/scripts/tlfiv5 (article «caricature», consulté le 2 septembre 2013). 
métamorphose, qui rappelle surtout le texte de Kafka dans la mesure où, derrière les différents visages de l'homme, c'est un animal étrange qui se dessine.

Il ne s'agit pas du lieu commun de la «bête politique », périphrase employée d'ailleurs par François Delacroix avec une nuance d'admiration, dont l'objet est aussitôt ramené à sa dimension humaine à travers l'expression : "l'homme qui a trois idées à la minute » (p.38). Il y a chez Karim Maoudj une métaphore filée qui sous-tend l'ensemble du discours et qui trouve son apogée en synthèse finale : «commence alors à prendre corps une autre créature » (p.172), commentaire signalant l'aboutissement d'un phénomène de diabolisation au sens de Ruth Amossy et Roselyne Koren :

La diabolisation est présentée comme une attaque outrancière effectuée sur le mode de la violence verbale, comme un appel à la peur et à la haine, et comme une tentative d'ostracisme, ressortant des domaines qui explorent le discours polémique, l'argument ad baculum et l'argument ad hominem. (Amossy, Koren, 2010 : 224)

Cette chronique d'une métamorphose annoncée débute en fait dès les premières pages de l'ouvrage et suppose à la fois une mise en cause de la raison du plus fort et une accumulation d'attaques personnelles. Régulièrement, Georges Frêche se transforme, aux yeux du lecteur de Karim Maoudj, en «jeune loup» (p. 20) menaçant la «bergerie socialiste», qui «gueule» (p.32) ou «hurle» (p.38), en «vieil aboyeur» (p.93) dont on souligne la «brutalité» (p.146), qui «ne manque jamais d'estomac» (p. 149) et est toujours prêt à asséner «des “coups" qui sont, la plupart du temps, le fruit de son flair» (p.173). En fin de parcours, le loup aux multiples facettes, dont on apprend que c'est un « grand timide» (p.179), se révèle soudain tortue («c'est la carapace qu'il s'est donnée ») et se mue finalement, à la toute dernière phrase du chapitre 6, en albatros qui «observe, impuissant, ses vastes ailes se replier» (p.189). L'ambivalence des images animalières, empruntées à l'univers des fables ou des poèmes allégoriques, est à noter, mais elles convergent toutes dans une même direction : un processus de déshumanisation du président du conseil régional alors en exercice.

\section{Alain Rollat: image d'un «homme de la Renaissance»}

Dans cette caricature multiforme qui présente une sorte de monstre correspondant à l'illustration en couverture du livre de Karim Maoudj, Alain Rollat ne reconnaît pas Georges Frêche, connu, certes, pour ses excès mais aussi pour ses réalisations et son combat idéologique. Ainsi qu’il le dit lui-même, il va travailler, à plusieurs niveaux, sur les représentations du personnage en essayant de comprendre comment une telle vision, celle qui donne le titre à sa première partie, a pu s'imposer, « une image à massacrer » (p.15). Son enquête passe par le décryptage de séquences télévisuelles et l'analyse discursive d'articles de 
presse qui ont tous le même déclencheur: «l'affaire des harkis » qui débute au moment où Georges Frêche, lors d'une manifestation publique, s'en prend avec véhémence à deux de ses interlocuteurs en les traitant de «sous-hommes».

La teneur de ce terme terrible, les circonstances de son actualisation, les conséquences politiques d'une telle violence verbale sont passées au crible par Alain Rollat qui ne cesse de s'interroger et prend donc - ce qui peut paraître paradoxal - cet événement de parole lourd de connotations et d'implications pour point de départ de la réhabilitation de l'ancien maire de Montpellier. Georges Frêche, déshumanisé par l'interprétation de ses propres propos (tenus en février 2006) et par le livre de Karim Maoudj (paru en avril 2007), va peu à peu redevenir un être humain à part entière sous la plume d'Alain Rollat. Dès l'ouverture du premier chapitre, il signale clairement que «le Georges Frêche dont l'image est livrée à l'opinion publique est un personnage agressif, grossier, violent, injurieux, méprisant bref indigne de sa charge élective» (p.15) et consacre la totalité du deuxième chapitre à démonter l'équation qui en découle: de par son langage, de par ses idées, de par sa haine, Georges Frêche est à gauche l'équivalent du chef de file de l'extrême-droite, Jean-Marie Le Pen. Dénonçant cette «fausse symétrie» (p.63), la première moitié du texte met en lumière un portrait de Georges Frêche qui fait écho à celui dressé par Karim Maoudj. Mais c'est pour mieux en retirer, au fil des deux derniers chapitres, les couches successives et montrer finalement, en analysant les articles de presse évoquant l'ancien maire de Montpellier sur les trente dernières années, une photographie qu'Alain Rollat juge plus conforme à l'original, basée non pas sur l'actualité déformante mais sur la durée.

C'est ainsi qu'il présente d'abord, utilisant un des vocables de son collègue, «un monarque désorienté», « un homme meurtri par un profond sentiment d'injustice» (p. 83), puis, dans le but de donner « une image juste, dans ses ombres comme dans ses lumières» (p. 85), une succession de portraits empruntés à Karim Maoudj et François Delacroix et à plusieurs confrères de la presse écrite. Les contrastes annoncés sont bien présents jusqu'aux deux derniers paragraphes, intitulés respectivement «Le Magnifique! » et « Le Tolérant». Les guillemets sont d'origine et indiquent qu'Alain Rollat résume alors l'enthousiasme perçu dans les discours analysés, antérieurs aux récents scandales. Mais, de manière certes implicite, il finit par s'approprier ces qualificatifs dans la lettre ouverte qu'il adresse à Georges Frêche :

L'image souveraine vous a déclaré inapte. Elle vous a assassiné et tout autre que vous serait mort. [...] Si vous n'aviez pas été un insupportable bulldozer, provocateur, colérique, tyrannique, vous n'auriez pas été le bâtisseur visionnaire de la «Florence du xxı e siècle». (A. R., p. 158-159)

Avec cette conclusion, non seulement Alain Rollat parachève le processus de réhabilitation, voire de réhumanisation de Georges Frêche, grâce à un portrait 
admiratif mais contrasté qui met en valeur la complexité de cette personnalité jugée hors norme et lui confère les lettres de noblesse d'un Laurent de Médicis, mais il inverse aussi le processus de déshumanisation en comparant, dans le titre du dernier chapitre de son livre, à une «meute» (p.121) les journalistes ayant renoncé à leur indépendance.

Ce corpus, extrêmement dense et varié derrière son apparente homogénéité, se caractérise finalement par son aspect multidimensionnel. Sur le plan discursif tout d'abord : la catégorisation générique est rendue extrêmement malaisée par la mise en jeu d'une polyphonie de genres journalistiques dont chacun «est adapté pour rendre un son particulier. Le reportage, c'est la vie. Le portrait, c'est l'épaisseur humaine. L'interview, c'est la riche sonorité d'une voix. L'enquête, c'est la clarté de la démonstration » (Mouriquand, 1997, p. 55). Ces différentes caractéristiques se retrouvent au sein de notre corpus dont les ouvrages ont pour point commun la trame narrative. Ils nous proposent des récits chargés d'intertextualité et d'effets de proximité, certes, mais qu'il est difficile de préciser davantage : ils racontent la carrière d'un personnage, à partir de sources multiples, pour en faire ressortir une image, afin que chaque lecteur puisse se forger sa propre représentation de l'homme dit à travers ces mots. En ce qui concerne Georges Frêche, l'aspect multidimensionnel fonctionne également au niveau politique : le lecteur des deux ouvrages principaux de notre corpus découvre un élu local ayant la stature d'un homme politique national non pas par ses fonctions ou ses actions, mais par la médiatisation abondante de ses propos. Pour Karim Maoudj, la dimension nationale de Georges Frêche n'explose qu'en fin de carrière, suite à ses débordements verbaux, voire ses dérives idéologiques, car les autres "affaires" " tout au long de son règne, n'ont jamais suscité le moindre intérêt des médias nationaux» (p.10). Pour Alain Rollat, cette dimension est mise en évidence bien plus tôt, dès le début des réalisations comme Antigone qui propulse Montpellier au rang des grandes métropoles françaises, faisant d'elle «une référence nationale et européenne » (p. 108) et c'est la mémoire interdiscursive ${ }^{12}$ de la presse qui est à mettre en cause, ses choix de focalisation à travers le temps. Concernant leur rapport à la sphère médiatique, tous deux en tout cas se placent dans une situation paradoxale. Bien qu'ils légitiment leurs écrits par leur position de professionnels de l'information, chacun se livre à une critique des médias. Pour Karim Maoudj, certains de ses confrères sont coupables de négligence ou de partialité; pour Alain Rollat, le reproche que l'on peut adresser aux journalistes renonçant à leur esprit critique est plutôt celui d'un acharnement, d'un emballement émotionnel.

12. «Le fonctionnement mémoriel est en effet complexe, fait à la fois de rappels intentionnels ou non, et d'oublis sémantiques partiels des sens, des situations et des événements. Se pose également, dès qu'on parle de faits du monde, la question de savoir sous quelles traces ils sont mémorisés.» (Moirand, 2007, p.115) 
La complexité est donc au rendez-vous de ces productions discursives en écho. Serait-ce le lot du journaliste politique devenu écrivain qui, en changeant de statut, transforme également son regard, ses stratégies énonciatives, son envie d'écrire et sa manière de dire? S'inscrivant dans une double démarche informative et argumentative, il dépasserait les normes imposées au reporter ou à l'éditorialiste pour développer, de manière intriquée et personnalisée dans la démarche et dans le style, une narration des faits davantage apparentée à un commentaire permanent, une prise de position non seulement autorisée par un genre où l'indépendance est de rigueur mais également clairement inscrite dans le contrat de lecture. Que ce soit en effet au niveau du rapport aux sources, dans la mise en jeu des échos intertextuels ou encore dans le choix des représentations, le livre permet au journaliste affranchi des contraintes imposées par la presse d'assumer une posture d'évaluateur, aussi bien vis-à-vis de l'objet étudié que vis-à-vis de la médiatisation de cet objet.

\section{Références}

ADAM Jean-Michel, 2001, "Genres de la presse écrite et analyse de discours», Semen, no 13, p. 9-16, en ligne: http://semen.revues.org/2597

AGnÈs Yves, 2002, Manuel de journalisme. écrire pour le journal, Paris, La Découverte. Amossy Ruth, 2000, L'argumentation dans le discours, Paris, Nathan Université.

- 2010, La présentation de soi. Ethos et identité verbale, Paris, PUF.

Amossy Ruth, Koren Roselyne, 2010, «La "diabolisation" : un avatar du discours polémique au prisme de la présidentielle 2007", Au corps du texte, D. Denis et al. éd., Paris, Champion, p. 219-236.

BOYER Henri, 2003, De l'autre côté du discours, Paris, L'Harmattan.

Charaudeau Patrick, 1997, Le discours d'information médiatique. La construction du miroir social, Paris, Nathan.

FoulQUIÉ Paul, 1978, Vocabulaire des sciences sociales, Paris, PUF.

KOREN Roselyne, 2004, "Argumentation, enjeux et pratique de l'“engagement neutre”. Le cas de l'écriture de presse », Semen, n¹7, p.19-40.

LE BART Christian, 2005, "La proximité selon Raffarin », Mots. Les langages du politique, $\mathrm{n}^{0} 77, \mathrm{p} .13-28$.

Maingueneau Dominique, 2005, Analyser les textes de communication, Paris, Armand Colin.

MoIRAND Sophie, 2007, Les discours de la presse quotidienne. Observer, analyser, comprendre, Paris, PUF.

MOURIQUAND Jacques, 1997, L'écriture journalistique, Paris, PUF.

RingOOT Roselyne, RochaRd Yvon, 2005, "Proximité éditoriale. Normes et usages des genres journalistiques », Mots. Les langages du politique, $n^{0} 77$, p. 73-90.

Trésor de la langue française informatisé, en ligne : http://atilf.atilf.fr/dendien/ scripts/tlfiv5. 
ÉLÉONORE YASRI-LABRIQUE

\section{Corpus}

DeLACRoIX François, 2007, Georges Frêche, la sagesse de la démesure, Céret, Alter Ego. MAOUDJ Karim, 2007, Georges Frêche, grandes heures et décadence, Paris, Éditions de Paris - Max Chaleil.

Rollat Alain, 2008, L'Assassinat raté de Georges Frêche, Sète, Éditions Singulières. 